\title{
Primauté technologique ou antériorité sociale dans la mesure des mouvements sociaux? Le cas du Sénégal et de la Tunisie
}

Popular movements in Africa: Technological primacy or social anteriority? Case studies of Senegal and Tunisia

\section{Benoît TINE et Mohamed-Ali ELHAOU}

\section{OpenEdition Journals}

Édition électronique

URL : http://journals.openedition.org/ctd/1697

DOI : $10.4000 /$ ctd. 1697

ISSN : 2491-1437

Éditeur

Chaire Unesco Pratiques émergentes en technologies et communication pour le développement

Édition imprimée

Date de publication : 12 novembre 2015

Référence électronique

Benoît TINE et Mohamed-Ali ELHAOU, «Primauté technologique ou antériorité sociale dans la mesure des mouvements sociaux ? Le cas du Sénégal et de la Tunisie », Communication, technologies et développement [En ligne], 2 | 2015, mis en ligne le 10 juin 2020, consulté le 22 juillet 2020. URL : http:// journals.openedition.org/ctd/1697; DOI : https://doi.org/10.4000/ctd.1697

Ce document a été généré automatiquement le 22 juillet 2020.

Communication, technologies et développement 


\section{Primauté technologique ou antériorité sociale dans la mesure des mouvements sociaux? Le cas du Sénégal et de la Tunisie}

Popular movements in Africa: Technological primacy or social anteriority? Case studies of Senegal and Tunisia

Benoît TINE et Mohamed-Ali ELHAOU

\section{Introduction}

1 La période préélectorale au Sénégal, plus précisément les années 2011 et 2012 ont été imprégnées par une contestation violente du régime d'Abdoulaye Wade - âgé de 85 ans et qui voulait rempiler pour un troisième mandat - par une jeunesse déterminée. Sous la houlette de rappeurs, dont la production est connue sur les médias numériques tels que You Tube, ces jeunes se sont réunis autour de "Y en marre ", un mouvement qui s'est illustré lors des émeutes de juin 2011 et lors du premier tour de l'élection présidentielle de 2012, avec comme leitmotiv : « Wade, dégage !».

2 En même temps, l'élan révolutionnaire commence à surgir en Tunisie à partir des mouvements de Redeyef en 2008, par la suite de ceux de Ben Guerdane au début de l'année 2010 et s'étend à l'échelle nationale au moment où Mohamed Bouazizi s'immole par le feu le 17 décembre 2010 (Chouikha, Geisser, 2010).

3 Pour construire notre argumentaire sur le Sénégal, nous avons utilisé un certain nombre de données. En effet, les événements ayant précédé le départ du président Abdoulaye Wade, au pouvoir depuis l'Alternance de l'an 2000 et qui voulait rempiler pour un troisième mandat, sont appréhendés d'abord à partir d'un corpus relatant ces mouvements sociaux, la littérature sociologique classique permettant de comprendre ceux-ci : il s'agit surtout de chercheurs tels que Dimé, Savané, Tine, Sall, Sarr ; puis 
nous avons eu une dizaine d'entretiens avec les protagonistes : bloggeurs, rappeurs, membres de la " société civile $»^{2}$ etc., exploités en partie dans une étude récente (Tine et Sall, 2014) ; enfin, nous avons analysé les discussions, conversations et interactions sur les réseaux sociaux : Facebook, You Tube. Ce matériau nous permet d'étudier les faits du 23 juin 2011, ayant consacré l'éveil citoyen ou ce qu'on a appelé le NTS (nouveau type de Sénégalais) (Savané, Sarr, 2012). Aussi, le cas sénégalais est jalonné par des interprétations de situations de conflits directs opposants des " y'en-amarristes " à l'image de journaliste tel que Fadel Barro, de rappeurs tel que " Fou malade ", de scientifiques tel que le Pr Malick Ndiaye et un régime en fin de règne incarné par la présidence de Wade et son propagandiste Farba Senghor.

4 Notre recherche sur le terrain tunisien s'appuie, elle, sur une revue de la littérature scientifique après les événements du 14 janvier 2011, notamment les écrits ayant été produits au moment de ces événements. Le but est de dresser un panorama partiel permettant de saisir comment se construit le récit scientifique par rapport à des objets de recherche que sont les mouvements sociaux d'un côté et les nouvelles organisations par la technologie de l'autre. Il importe de préciser que le matériau cité dans le corps de cet article ne se fonde pas sur des passages glanés çà et là. Ce sont bien au contraire des citations choisies minutieusement suivant qu'elles versent ou pas dans l'éloge des logiques sociales d'une part ou bien des potentialités encore incomprises ou inexplorées des TIC. En d'autres termes, nous étudions depuis 2011 la littérature scientifique portant sur l'articulation des TIC et des mouvements sociaux. Pour essayer de rendre compte de cette production scientifique, notre tâche consiste dès lors à repérer les arguments, saillants ou suggérés, à partir desquels les auteurs prennent position de manière plus ou moins nette entre le phénomène technologique et le phénomène social. Notre démarche est heuristique. Elle consiste à proposer une perspective médiane interrogeant les schèmes explicatifs dominants et qui ne sont pas forcément dans le déterminisme. Ce positionnement épistémologique, qui sans faire de théories, pointe du doigt une mutation en cours spécifique au contexte africain. Cette mutation repose sur deux aspects essentiels à savoir que les relations de face-à-face, la transmission et la " raison orale " (Diagne, 2006) des récits se conjuguent désormais avec des formes de communication à distance qui privilégient l'immédiateté et la raison utilitaire. Ces formes de communication que les TIC amplifient modifient en profondeur la façon de faire de la politique et le rapport au vivre ensemble (Voirol, 2005), notamment dans des contextes africains marqués par l'inachèvement de l'État (Mezghani, 2011).

5 La triple question de cette recherche est la suivante : les TIC sont-elles exclusivement une " étincelle " réveillant un mouvement social sous-jacent, dormant dans deux contextes africains ? Ou bien doivent-elles être saisies comme des instruments de participation et d'organisation qui favorisent la formation d'une " intelligence collective » (Levy, 2002) ? Dans les deux sens, les médias numériques conduisent-ils à une « cyberdémocratie »?

6 Ce texte est foncièrement épistémique. Tout au long de ce travail nous essayons de remettre en question voire de critiquer le paradigme partiel et partial de l'éloge du social d'un côté et de l'éloge de la technique de l'autre. En second lieu, ce texte propose d'analyser ensemble, ce qui est un effort méthodologique non négligeable, deux terrains qui montrent la diversité d'une Afrique souvent traitée comme étant un bloc homogène par les chercheurs des pays anglo-saxons et de manière très clivée par les 
chercheurs européens (Kane, 2012). Ces derniers distinguent bel et bien zone arabe et zone Afrique noire. En ce sens, l'un des apports de cet article est d'élaborer « un travail sur le lien » (Lincoln, 2010).

\section{Le paradigme des TIC VS le paradigme des logiques sociales}

7 La rhétorique du " printemps arabe " se loge au sein du paradigme déconstruit par Alain Kiyindou de l'Internet comme un dispositif émancipatoire des peuples en développement et de l'avènement de la "société de l'information» (Kiyindou, 2009). Pour les analystes du " printemps arabe ", en tant qu'appellation principalement politique, celle-ci a été source de divisions entre chercheurs. Il y a, en l'occurrence, ceux qui déconstruisent ce même " printemps » et mettent par là même l'accent sur une exaspération sociale de longue haleine (Cardon et Granjon, 2013). Cette frustration, dont la provenance serait les tréfonds des régions pauvres et qui a été relayée par médias, citoyens et journalistes, émanerait donc des émeutes de Redeyef de 2008, des insurrections de Ben Guerdane en 2010 ainsi que des multiples mouvements sociaux se poursuivant depuis le 14 janvier 2011 en Tunisie. Contrairement à cette lecture, d'autres chercheurs œuvrent pour la promotion du rôle des TIC en essayant de découvrir ses potentialités émancipatrices.

\subsection{Les détracteurs du rôle des TIC comme source d'un « printemps arabe »}

8 Parmi les détracteurs des TIC comme source d'un dit « printemps arabe ", Sarah Joseph place l'antériorité sociale comme un socle explicatif inaliénable. L'auteure considère en effet que les logiques sociales comme se situant à l'avant-garde d'un désenchantement citoyen ; lequel serait la conséquence d'une mondialisation à la fois agressive et inachevée sans cesse productrice de mouvements sociaux (Della Porta et Kiersi, 1998 ; Pouligny, 2001). Dans cette perspective, les supports numériques ne sont qu'une alternative faible et provisoire favorisant la mise en scène d'un esprit vindicatif spectaculaire sans plus. En ce sens, les artefacts numériques poursuivent autrement le rôle des médias traditionnels plus qu'ils ne changent les rapports de domination en place. Certains chercheurs effacent complètement le rôle des TIC dans la production des mouvements sociaux. Ils privilégient l'hypothèse selon laquelle l'étincelle technologique n'eut tout simplement pas lieu et que celle-ci n'existerait qu'une fois insérée dans une logique d'engagement social.

"Révolution 2.0 ", écrivent Riadh Ferjani et Tristan Mattelart, " révolution Facebook ", " révolution Twitter »: de quels qualificatifs n'a-t-on pas usé pour mettre en évidence la participation des technologies de communication à la chute des régimes de Ben Ali ou de Moubarak ? Au risque d'ériger les vertus démocratiques d'Internet en passe-partout trop commode. En rupture avec ces visions, au fort parfum de déterminisme technologique, nous avons intitulé ce dossier «Les révolutions 2.0 n'ont pas eu lieu », pour souligner le fait que ces bouleversements politiques, loin d'être le résultat de la prétendue force libératrice des réseaux, sont d'abord et surtout l'aboutissement de mobilisations sociales » (Ferjani et Mattelart, $2011: 70$ ). 
Ces deux chercheurs insistant sur l'antériorité des logiques sociales considèrent en effet lesdites " révolutions arabes " un " réveil arabe " voire un soulèvement. Ces mouvements sociaux traduiraient donc une frustration sociale (Gurr, 1970) de longue portée et n'ont pas attendu donc les médias numériques (Facebook, Twitter) pour prendre forme. Sous cet angle minimisant la primauté technologique, les potentialités numériques ne modifient pas le rapport entre gouvernants et gouvernés et encore moins elles n'assurent l'accès à une Révolution. En d'autres termes, les technologies modernes par leurs simples usages ne produisent pas, selon ce schème analytique, une rupture et ne modifient pas les anciennes pratiques de coercition qui marquent les pays africains de manière générale (Allal et Geisser : 62-67). Dans la lignée d'Allal et Geisser, certains chercheurs considèrent les TIC comme ouvrant la porte à de " nouvelles formes de dictature » (Evgeny, 2012).

\subsection{Les promoteurs du pouvoir émancipateur des TIC}

10 Les détenteurs de cette thèse considèrent les technologies numériques comme composantes essentielles des dynamiques sociales modernes en matière d'innovation dans l'expression. Ce prisme, apparu une première fois au début du développement de l'Internet à partir de la moitié des années 1990, est revenu avec force après les innovations ayant conduit au " web 2.0 ». (Neveu, 1994: 72). Les TIC, selon cette explication, ne se diluent pas dans les mouvements sociaux, mais les provoquent en faisant tomber certains obstacles liés à une culture traditionnaliste et rigide. Les TIC ouvrent dès lors de nouvelles perspectives d'organisation, d'expression et d'engagement (Castells, 2002). Elles appliquent l'adage prôné par Wael Ghonim ${ }^{3}$ qui disait : « si tu veux libérer la société donne-lui l'Internet ». Dans la même veine, les TIC vont même être envisagées comme pourvoyeuses d'une dynamique non seulement de " participation " jusqu'à aujourd'hui formalisée (information, consultation, concertation, codécision), mais " expressiviste ", " engageante » (Monnoyer-Smith, 2011) voire de capitalisation de relations à travers la création d'une communauté virtuelle et la diffusion des informations servant à déstabiliser le système en place.

11 Certains chercheurs considèrent les scènes filmées par cette communauté via des téléphones portables comme participant à l'émancipation des peuples. D'autres vont mettre l'accent sur le fait que les médias numériques ont un rôle de " caisse de résonance » (Chouikha, Gobe, $2011:$ 219-226) d'où émane la voix d'internautes le plus souvent marginalisés. Aussi, ces technologies en propageant la dimension dénonciatrice au régime autoritaire ont ouvert la possibilité à la diaspora de participer à la dénonciation collective (Lecomte, 2009) par la publication d'informations tues au sein des desks des médias traditionnels. Avec cette même conception, Anita Breuer, dans une étude examinant les comportements des usagers dans les médias numériques tunisiens, renvoie le "printemps arabe » essentiellement aux artefacts techniques. Ceux-ci selon ses analyses " permettent " de contourner les frontières socioéconomiques et géographiques (Breuer, 2012 : 25-26). Bien plus, certains chercheurs plaident pour le fait que les TIC ouvrent une triple brèche : une augmentation de la fluidité et de la liberté des échanges, la construction progressive de la "sphère de l'immatériel » (Moulinier-Boutang, 2007) et des transformations de l'action humaine faisant passer celle-ci d'un " simple calcul » vers le « sentiment d'exister » (Alter, 2010 : 113). Ils mettent en exergue ces artefacts comme sources de partage de sentiments et 
d'émotions engendrant ainsi un 'cognitive surplus' se produisant dans un environnement de " démocratie Internet " (Cardon, 2010). Les technologies selon Clay Shirky (Clay, 2010) produisent une rupture « radicale » et transforment foncièrement les usagers de la technologie de simples consommateurs passifs en collaborateurs informés et actifs agissant sous le modèle de l'acteur-réseau (Latour, 1997)

\subsection{Le pouvoir émancipateur se loge exclusivement dans les logiques sociales : le cas sénégalais}

12 Selon les analystes du cas sénégalais, les mouvements de protestation citoyenne et pacifique ont véritablement commencé à la suite de coupures d'électricité intempestives et quasi-faillite de la société nationale d'électricité (Sénélec), hausse des prix des denrées de première nécessité, inondations, chômage de masse entraînant un exode massif de ces jeunes vers Lampedusa, la fameuse île italienne, à bord d'embarcations de fortunes (Daniel, 2008), gabegie et corruption, scandale financier et immobilier etc. (Cissokho et Sidy, 2011), ayant même entraîné la radicalisation de certains imams ${ }^{4}$, ont connu leur apogée le 23 juin 2011.

13 En effet, ce jour-là, était prévu à l'Assemblée nationale du Sénégal, le vote d'une loi modifiant le mode de scrutin de l'élection présidentielle de 2012. Ce projet de loi devait permettre l'élection simultanée du président et du vice-président, au premier tour, avec seulement $25 \%$ des voix. Ce énième tripatouillage de la constitution avait pour but d'officialiser l'accaparement de tous les leviers du pouvoir par Karim Wade, le fils du Président de la République et assurer ainsi la réélection de " $\mathrm{pa} \mathrm{bi}^{5}$ »lors des élections présidentielles en février 2012. Mais rien ne se passera comme prévu.

14 Malgré cette élucidation politique technocratique, une expression ironique fait florès au sein de la population sénégalaise : Rewni daffa metti ${ }^{6}$. Cette expression montre au grand jour à quel point la population est déçue d'un État qui abandonne ses citoyens les plus fragiles à la misère et à la pauvreté à l'heure de la pénétration de l'économie marchande. Dans cette configuration, une grande majorité de la population et surtout les jeunes dans ce pays considéré comme " la vitrine démocratique » en Afrique, est livrée au pouvoir des marabouts (Loum, 2014 : 25-38). Suivant cette lecture, le primat $\mathrm{du}$ social voire du religieux est saillant. Dans un contexte de contraintes socioculturelles, les manifestations qui eurent lieu en 2011 sous la houlette de « Y en a marre " sont nées d'un ras-le-bol généralisé : des coupures intempestives de l'électricité, des inondations récurrentes, la gabegie et la corruption érigées en mode de gouvernance, des grèves endémiques dans l'éducation nationale et l'enseignement supérieur, le néo-patrimonialisme Étatique (Médard, J-F, 1991) et des chômeurs résignés. Ce cri de cœur d'une jeunesse sénégalaise désemparée n'est pas nouveau en soi et rappelle des mouvements tels que " la génération boul-falé » sous le régime du Président Abdou Diouf ${ }^{7}$ (1980-2000). Ces postures consacrent des dispositifs incorporés et s'inscrivent dans une tradition de mobilisation citoyenne et d'engagement politique qui caractérise la jeunesse sénégalaise depuis quelques décennies.

15 À la différence de ces dernières, la contestation sociopolitique de 2011 par « Y en a marre » a été relayée par les médias traditionnels via la presse écrite et les radios et timidement par les médias numériques ; parce que nécessitant au préalable un accès d'abord à l'électricité puis à Internet. Cette infrastructure numérique tarde d'ailleurs à se démocratiser quantitativement et qualitativement. Néanmoins, la technologie est 
bien dans son rôle quand elle accompagne l'avènement d'un NTS (nouveau type de sénégalais) et l'émergence d'un changement social en servant de canal de transmission. Aussi, à l'examen du contexte sénégalais, la thèse de la primauté des logiques sociales et l'insertion des TIC en son sein semblent s'imposer. En effet l'émergence des réseaux numériques ne génère pas de mouvements sociaux. D'ailleurs, le taux de pénétration d'Internet reste encore faible avec $17,5 \%$ en 2013 et le nombre d'utilisateurs de Facebook était estimé à la même date à 730940 sur une population totale de 13500000 .

Alain Touraine (1973 : 361-363), dans sa construction d'une sociologie du conflit abonde dans le même sens et affirme que la société se construit à travers la lutte des mouvements sociaux qui empruntent un long processus en vue d'un changement. On peut donc comprendre le mouvement " $\mathrm{Y}$ en a marre " quand il appelle de tous ses vœux l'avènement d'un NTS : nouveau type de sénégalais (Dimé, 2013).

$\mathrm{Au}$ Sénégal, les logiques sociales ont été décisives de facto puisque le régime du président Wade, à partir de 2000, a entrainé ce pays sur des pentes dangereuses à coups de bricolages incessants de la Constitution exacerbant les écarts entre le centre et la périphérie, les riches et les pauvres, bref, les inégalités entre citoyens. Autrement dit, en réduisant et en libéralisant crescendo les biens communs à tous au grand dam de ceux qui n'arrivent pas à s'adapter à un mode de production fondé sur le modèle de The winner takes the most ${ }^{8}$.

Le cas sénégalais renvoie donc à la thèse suivant laquelle les TIC sont solubles entièrement dans les logiques sociales. Dans cette lignée, le développement des médias numériques ne saurait conduire, de la façon que nous observons aujourd'hui, à des révolutions sociales sui generis. Ces nouveaux médias numériques, suivant le paradigme de l'antériorité sociale dans la production des mouvements, concrétisent la prophétie du " bluff technologique » (Ellul, 1977) voire « une théologie libertaire du réseau » (Musso, $2011: 3$ ). Il n'en demeure pas moins que cette perspective avantageant les logiques sociales peut occulter l'innovation et négliger la technologisation (Mœglin, 2005) du mouvement social et ses nouvelles implications en matière d'engagement dans la sphère publique.

\section{Technologisation du mouvement social et les enjeux en lice}

19 L'approche médiane c'est-à-dire communicationnelle que nous proposons dans ce point va dans le sens que le mouvement social est un phénomène qui se technologise davantage. Dire cela, c'est dépasser en l'occurrence la bipolarisation, la plupart du temps stérile, entre le social d'une part et la technique de l'autre (Latzko-Toth, 2002 : 174). C'est aussi rompre avec une idée courante suivant laquelle l'individu est aliéné, voire solitaire, devant l'écran de son ordinateur. De ce fait, la focalisation sur la technologisation du mouvement social nous montre bel et bien que le simple usager en Afrique une fois connecté est pris bien au-delà du dispositif technique, et est naviguant dans son propre univers de références. Celui-ci lui transmet des idées et des façons d'agir dans une ambiance ludique, spontanée (Durampart, 2013 : 222) et sans assignation faisant émerger « de nouvelles formes du social » (Jouët, 2011 : 52). Dans ce sillage, l'appropriation des médias numériques peut être envisagée, en partie, comme une action dans un lieu (De Certeau, 1975) immatériel (Gorz, 2003). Endroit décuplant et mettant en avant des pratiques et des actions communicationnelles individuelles qui 
visent la mise en visibilité des "déviances » (Becker, 1963) au sein d'un système politique instrumentalisant les médias classiques.

Certes, nul ne fait la révolution en étant assis devant son ordinateur, mais le fait d'être branché n'est nullement significatif d'un acte d'isolement. L'individu dans cette situation peut en l'occurrence concrétiser des idées déviantes dormantes avec les nouvelles possibilités offertes au sein de l'arène numérique. Les dispositifs techniques « hétérogènes » ont donc un ancrage socioculturel comme le confirme Remy Rieffel et " affectent tous les secteurs de l'activité humaine » (2014:262).

21 C'est à ce titre que dans les deux pays africains, le Sénégal et la Tunisie, nous repérons une propension à la reconnaissance par le biais de la technologie voire même une recherche de solution in extremis de la part de jeunes citoyens dans un continent qui n'arrive pas à les contenir ou du moins qui ne leur offre pas assez d'issues d'avenir en matière d'emploi (Tine, 2014) et de réalisation de soi.

\subsection{Démocratie Internet ou anarchie banalisée?}

22 Avec la dissémination de la technologie dans la sphère politique, plutôt que de conduire vers une dialectique nationale constructiviste, les formes de communication dans la sphère virtuelle en Afrique se prêtent en grande partie à de la non-communication, à l'échange d'invectives et à la zizanie généralisée. Mais en même temps, l'heure des " monologues interactifs " (Flichy, $2008:$ 163) sonne le glas du monopole du discours dans les sphères officielles à l'œuvre dans les pays africains et montre en partie qu'avec Internet, tout le monde peut s'exprimer; le droit à la parole se démocratise et se banalise. En effet, les technologies dans des contextes africains prépareraient de ce fait l'avènement d'une société conçue comme étant plus adhocratique ${ }^{9}$ et de moins en moins pyramidale et rigide. Les artefacts numériques mettent donc en commun les efforts de chaque usager au Sénégal au même titre qu'en Tunisie pour une augmentation de la transparence. Celle-ci devient plus une cause voire une campagne de militantisme sur le net qu'une réelle question de débat d'idées au sein d'un espace public idéalisé. Ce fait est saillant à l'observation des activités du microblogging et les discussions en ligne sur des forums dans ces deux pays.

23 Antoní Gutiérrez-Rubí admet dans cette même lignée que la pratique du microblogging, étant une des potentialités offertes par les technologies numériques « maintienne la création de l'esprit de la révolution » (Gutiérrez-Rubí, 2011 : 17-21) par la transmission des émotions de manière instantanée et par l'alimentation constamment des « guerres d'injures " évoquées par Flichy. Le processus de la numérisation matérialise donc le discours sur l'évolution de l'activité politique en faisant exister différents modèles politiques (Wright, 2007 ; Monnoyer-Smith, 2010 ; Noveck, 2008).

En ce sens, le concept même de démocratie serait en train d'évoluer (Dahlberg, 2011) en faisant muter les formes de la relation gouvernants/gouvernés (Coleman et Shane, 2012) vers la prise en considération des espaces de la technologie comme des territoires à la fois de veille et de l'expression sensible de l'opinion publique. Cette explication, qui s'inscrit au sein même de l'approche médiane que nous proposons, montre que le parcours médiatique des sursauts populaires se conjugue de plus en plus avec la spectacularisation numérique visible en partie à travers le " web militant " (Reiffel, 2014 : 253). Les vidéos postées sur YouTube ou Dailymotion emplissent en un temps record les réseaux numériques. Ensuite, des révoltes et manifestations, surtout de 
jeunes qualifiés " d'enfants de Facebook », en ont découlé. Depuis les blogs, Twitter, You Tube et Facebook, des vidéos amateurs relaient massivement les manifestations qui ont eu lieu. Elles révèlent la répression qui s'en est suivie. Des répressions ensuite diffusées par Al Jazeera, France 24 suscitant l'indignation et mobilisant toute la population. Si les jeunes, en tant que groupes marginaux en Afrique, décident de s'approprier le domaine de l'information c'est parce les médias numériques autorisent des prises de parole qui dépassent le récit journalistique dominant. Ces mêmes médias brisent la tradition flatteuse en cours dans le fonctionnement bureaucratique des médias de masse aussi bien en Tunisie qu'au Sénégal. Dans cette perspective, les dispositifs numériques favorisent la mise en coordination d'actions collectives et l'élaboration des cognitions identiques qui se propagent de manière réticulaire c'est-àdire en réseau ; et ce aussi bien de manière visible (médias numériques) qu'invisible (des courriels). De part son architecture orientée vers l'interaction et l'exposition de soi, le web serait source de la formation d'une «boule de neige » numérique (Sunstein, 2001) qui renflouerait la dialectique entre l'information marginale (Ben Amor, 2013 : 5) circulant sur le réseau et l'information officielle acculée désormais sur son propre terrain.

\subsection{L'approche médiane montre la fétichisation du mouvement social}

En partant du cas sénégalais, force est de constater dans la littérature consacrée que les technologies ont été fétichisées. En d'autres termes, les chercheurs (Savané, Sarr, 2012) leur ont fait jouer un rôle qu'elles n'avaient pas alors qu'en Tunisie. En se dotant de posture médiane, il est à noter que les plateformes numériques ne sont pas sources de mouvements sociaux, mais plutôt de fétichisation du changement social c'est-à-dire de mise en visibilité qui cherche non seulement le vivre ensemble, mais aussi le voir ensemble (Voirol, 2005 : 99). Ces plateformes rajoutent une médiatisation supplémentaire en donnant plus de proximité à ces mouvements. Elles accroissent de facto la dimension subliminale et spectaculaire du mouvement social en lui donnant immédiatement des résonances mondiales comme ce fut le cas avec ledit " printemps arabe ». Parallèlement, il serait tellement facile de dire : ça y est, le changement est là grâce à la e-démocratie voire grâce à une nuée de cyberactivistes. D'ailleurs, le réel africain est objectivement très loin de cet avènement euphorique de la démocratie. Le quotidien est bien encore très lourd à supporter pour des individus qui accèdent très difficilement à la citoyenneté; autrement dit à l'éveil citoyen ou à ce que l'on a appelé le nouveau type de Sénégalais (Savané, Sarr, 2012). Dans ce contexte de paupérisation de l'individu, la fétichisation du mouvement social est entreprise par de nouvelles stars de la sphère virtuelle (Elhaou, 2012 : 379-385). En cela ces stars se rapprochent des pratiques activistes et de l'environnement festif des branchés détaillés par Dominique Carré (Carré, 2008 : 7).

En suivant le raisonnement de Dominique Carré, même si les TIC ne révolutionnent pas le monde, elles introduisent néanmoins une véritable dynamique de changement social. Dans cette perspective notre approche communicationnelle nous autorise plutôt de suggérer la quête du " petit quart d'heure de célébrité » dont parlait Andy Warhol. En agissant et en réagissant sur Internet, l'internaute joue donc un rôle. Il endosse un costume d'acteur et verse dans une théâtralisation en se donnant en même temps et surtout du plaisir. Il ne ferait rien d'autre que de consommer ou tout simplement de se 
mettre en scène devant son écran d'ordinateur, nouvel " agora des temps modernes " (Tine, $2014:$ 16). De là à vouloir changer le monde, la frontière n'est pas si poreuse et l'exemple sénégalais l'atteste (Dimé, 2013). Certes les TIC donnent cette impression de force, de puissance et le sentiment de diriger et par conséquent de changer le monde, voire de donner une leçon au monde entier d'un coup de baguette magique; mais, à l'heure de la fétichisation des mouvements sociaux, la quête de notoriété en Afrique ne dépend plus seulement d'intermédiaires institutionnels, d'autorités légitimes (les pairs, les spécialistes à travers les procédures très codifiées), mais de l'écho que certaines actions politiques et sociales rencontrent dans les médias numériques. En ce sens, ces actions malgré leur courte durée cherchent des affluences au niveau des commentaires et de la capitalisation en matière du nombre de partages pour faire pression sur le système en place. De surcroit, à l'intersection de l'explosion technique et de la prolifération des revendications émerge la culture de l'exposition. Celle-ci est de plus en plus prégnante sur le web africain. En relation complémentaire avec le monde réel, cette culture part du principe que l'essentiel est de se faire remarquer que l'important n'est pas nécessairement d'être original ou créatif, mais d'être vu ; d'occuper l'espace médiatique et d'attirer l'attention par rapport à un projet individuel ou collectif.

\section{Conclusion}

Dans un contexte africain articulant deux situations différentes telles que le Sénégal (Afrique au Sud du Sahara) et la Tunisie (Afrique du Nord), au lieu d'avantager les logiques sociales au détriment de ce qui est qualifié de déterminisme technologique ; ou encore d'exagérer le rôle dit " révolutionnaire " des TIC en récusant les logiques sociales ; nous avons ébauché dans le troisième point de l'article une perspective médiane mobilisant une approche communicationnelle.

Selon cette perspective médiane, il est plausible d'aller dans le sens que les TIC ont bel et bien constitué durant les dernières années de la dictature de Ben Ali (Elhaou, 2010 : 47-77) - mais aussi pendant son règne - un dispositif de pouvoir et de surveillance, mais aussi des lieux virtuels pour élaborer en marge du récit officiel un vocabulaire contestataire.

Légitimer in fine ce double prisme mobilisant à la fois la primauté technologique/ sociale est une façon d'examiner le cas sénégalais et tunisien, comme des contextes où se produit du sens. L'étude de la littérature scientifique et des discussions sur les réseaux sociaux sénégalais que nous avons effectuée ainsi que l'interprétation des conflits opposant les " $\mathrm{y}$ en a marristes » au régime Wade (agonisant) permettent de repérer, mais aussi d'observer qu'une grande partie des mouvements d'action collective mise en place par des jeunes générations dans le continent africain expriment des revendications d'existence digne et de reconnaissance. Cette catégorie de jeunes comme nous avons montré, en conjuguant (ou en répartissant) activisme en ligne et activisme sur le terrain réclame dans sa critique et par le biais des slogans qu'elle mobilise le droit à l'existence sociale, publique voire en tant que citoyen du monde.

Nous avons explicité dans le point relatif à la fétichisation du mouvement social comment l'usage des TIC est une façon de faire connaitre et d'amplifier le registre de revendication et même l'intensité de la colère de la population à l'égard du système en place, mais cette colère se passe à travers une forme d'exposition culturelle et festive 
tel que le rap. Cette forme artistique a été un des moteurs du changement aussi bien en Tunisie qu'au Sénégal. Phénomène à suivre davantage.

\section{BIBLIOGRAPHIE}

BIBLIOGRAPHIE

ALLAL A., et VINCENT G., 2011, « Tunisie : 'révolution de jasmin' ou intifada? », Mouvements, n66, été, dossier « Printemps arabes : comprendre les révolutions en marche », pp. 62-67

ALTER N., 2010, Donner et prendre. La coopération en entreprise, Paris, La Découverte/Poche.

BALANDIER G., 2001, Le Grand Système, Paris, Fayard.

BEN AMOR, A., 2013, « Cyberdissidence tunisienne », Communication [En ligne], Vol. 32/1 | 2013, mis en ligne le 29 novembre 2013, consulté le 05 mai 2014. URL: http://

communication.revues.org/4707; DOI: 10.4000/communication.4707

BECKER H., 1963, Outsiders: Studies in the Sociology of Deviance, New York, the Free Press

BREUER A., 2012, “The role of social media in mobilizing political protest. Evidence from the Tunisian revolution", German Development Institute Discussion Paper No. 10/2012, Deutsches Institute für Entwicklungspolitik (DIE), ISSN 1860-0441. Disponible à l'SSRN: http://ssrn.com/ abstract=2179030 or http://dx.doi.org/10.2139/ssrn.2179030

CARDON D., 2010, La Démocratie Internet. Promesse et limites, Paris, Coédition Seuil - La République des idées

CARDON D., et GRANJON F., 2013, Médiactivistes, (Nouvelle édition revue et complétée) Paris, Presses de science po'

CARRÉ D., 2008, « Entre anonymat, visibilité internétique et publicisation. Les pratiques activistes du Clan du Néon », in Espaces de vie, espaces-jeux : Entre investissements ordinaires et mobilisations politiques, Actes du colloque international, Université de Rennes 2 - Crape-Eso, 5-7 novembre 2008, CD-ROM, pp.1-8

CASTELLS M., FERNÁNDEZ-ARDÈVOL M., QIU J. \& SEY A., 2006, Electronic Communication and Socio-Political Mobilization: A New Form of Civil Society, in Global Civil Society 2005/6, M. Glasius, M. Kaldor \& H. Anheier (eds.), Sage Publications, London, pp.112-131

CHOUIKHA L., et GEISSER V., 2010, « Retour sur la révolte du bassin minier. Les cinq leçons politiques d'un conflit social inédit », L'Année du Maghreb, VI, Paris, pp. 415-426

CHOUIKHA L., 2015, La difficile transformation des médias. Des années de l'indépendance à la veille des élections de 2014, Tunis, Éditions Finzi

CISSOKHO Th., SIDY, 2011, « Y'en a marre » Rap et contestation au Sénégal, Multitudes 3/ 2011, n 46

CLAY, Sh., 2010, Cognitive Surplus, How Technology Makes Consumers into Collaborators, New York, Penguin Books

JONES E-S., 2013, The Emergence of the Digital Humanities, NY, Routledge 
DANIEL S., 2008, Les Routes clandestines, Paris, Hachette

DE CERTEAU M., 1975, L'Écriture de l'histoire, Paris, Gallimard

DELLA PORTA D. et KRIESI H., 1998, « Movimenti sociali e globalizzazione », Rivista Italiana di sienza Politica, pp. 451-482

DIAGNE, M., 2006, Critique de la raison orale. Les pratiques discursives en Afrique noire, Paris, Karthala

DIMÉ M., 2013, « Y’en a marre ! Puis après ? - Le mouvement Y’en a marre au Sénégal : éphémère cri de colère ou dynamique juvénile d'affirmation citoyenne ?» Communication au 81ème du Congrès de l'Acfas : La jeunesse : moteur du changement en Afrique subsaharienne ? 6-10 mai 2013, Université de Laval, Québec

DURAMPART M., 2013, «L'expression des cybercitoyens entre continuité et ruptures face à une sociabilité quotidienne » in Sihem Najar (dir), Le cyberactivisme au Maghreb et dans le monde arabe, Paris, KARHALA et IRMC, pp. 217-226

ELHAOU, M-A., 2010, «La rhétorique du "Changement" comme dispositif d'innovation en Tunisie ", ESSACHESS -Journal for Communication Studies, Vol 3, N5, Toulouse, pp. 47-77

ELHAOU M-A., 2012, « La fabrique médiatique de la critique tunisienne entre innovation technologique et changement social » in Amsidder Abderrahmane, Daghmi Fathallah, Toumi Farid (dirs), Usages et pratiques des publics dans les pays du sud. Des médias classiques au TIC, Agadir, Université Ibn Zohr, 2012, pp. 379-385

ELLUL J., 1977, Le Système technicien, Paris, Calmann-Lévy

ETLING B., FARIS R. \& PALFREY J., 2010, "Political Change in the Digital Age: The Fragility and Promise of Online Organizing", SAIS Review, 30(2), pp. 37-49

EVGENY M., 2012, The Net Delusion: the dark side of Internet freedom, New York, Kindle Editions FERRIÉ J-N., 2003, « « Société civile », autoritarisme et globalisation des normes », Journal des anthropologues, $\mathrm{n}^{\circ}$ 94-95, pp.75-91

FLICHY P., 2008, « Internet, un outil de la démocratie ? », laviedesidees.fr, pp.1-16

FLICHY P., 2008, «Internet et le débat démocratique », Réseaux, 2008/4 n 150, pp. 159-185. DOI : 10.3166/réseaux.150.159-185

FLICHY P., 2010, Le sacre de l'amateur, Sociologie des passions ordinaires à l'ère numérique, Paris, Seuil, (coll.) La République des idées

GEORGE É., 2011, « Analyser les rapports entre société et technologie à partir et au-delà de l'opposition technologisme/sociologisme », Annabelle Klein et Serge Proulx (dir.), Communication numérique et lien social, Namur : Presses universitaires de Namur, pp. 65-82

GOBE É, et CHOUIKHA L., 2011, « La Tunisie virtuelle : caisse de résonance du mouvement protestataire ", in La force de la désobéissance : retour sur la chute du régime de Ben Ali, Armand Colin, coll. Revue du Tiers Monde, pp. 219-226

GORZ A., 2003, L'immatériel, Paris, La Découverte

GUTIÉRREZ-RUBÍ A., 2011, "Del storytelling al microblogging”, Cuadernos de Comunicación Evoca (4ํㅡㄹ Comunicación política 2.0), Madrid, pp. 17-21

GURR T., 1970, Why Men Rebel?, Princeton University Press, Princeton 
HAERINGER N., 2012, « Y’en a marre, une lente sédimentation des frustrations », Mouvements, Vol 1, n 69 , pp. $151-158$

ION J., (dir.), 2001, L'engagement politique, Saint-Etienne, Publications de l'Université

JOSEPH S., 2011, "Social Media, Human Rights and Political Change", Boston College International \& Comparative Law Review, Vol. 35, pp. 145-188

JOUËT J., 2011, « Des usages de la télématique aux Internet Studies », dans DENOUËL J., et GRANJON F., (dir.) Communiquer à l'ère numérique. Regards croisés sur la sociologie des usages, Paris, Presses DES MINES

KANE O., 2012, « Épistémologie de la recherche qualitative en terrains africains : considérations liminaires », Recherches Qualitatives, Vol. 31, (1), pp. 152-173

KIYINDOU A., 2009, Les pays en développement face à la société de l'information, Paris, L'Harmattan

LATOUR B., 1997, Nous n'avons jamais été modernes. Essai d'anthropologie symétrique, Paris, La Découverte; nouvelle éd

LATZKO-TOTH G., 2002, « Internet comme lieu de la socialisation », in JAURÉGUIBERRY F., PROULX S., (dir.), Internet nouvel espace citoyen?, Paris, L'Harmattan, 165-180

LECOMTE R., 2009, « Internet et la reconfiguration de l'espace public tunisien : le rôle de la diaspora », tic\&société [En ligne], Vol. 3, n 1-2|2009, mis en ligne le 12 janvier 2010, Consulté le 29 octobre 2013. URL : http://ticetsociete.revues.org/702 ; DOI : 10.4000/ticetsociete.702. (Dernière consultation, le 29 octobre 2013)

LECOMTE R., 2011, « Révolution tunisienne et Internet : le rôle des médias sociaux », L'Année du Maghreb, VII, pp. 389-418

LEVY P., 2002, Cyberdémocratie, essai de philosophie politique, Paris, Odile Jacob

LINCOLN Y. S., 2010, “What a long, strange trip it's been..." twenty-five years of qualitative and new paradigm research. Qualitative Inquiry, 16 (1), 3-9.

LOUM N., 2014, « Les médias et les marabouts au Sénégal : l'indépendance postulée face aux contraintes socioculturelles », in Chouikha L., et El Bour H., (dir.), Etre journaliste, aujourd'hui, à l'ère de la mondialisation de la communication. L'exemple des pays arabes et africains, Actes de colloque international 21-22 avril 2011, Institut de presse et des sciences de l'information (IPSI) et Konrad Adenauer Stiftung, Tunis, pp. 25-38

MAUSS M., 1973, « Essai sur le don, Forme et raison de l'échange dans les sociétés archaïques », Sociologie et Anthropologie, Paris, PUF, Collection Quadrige, pp. 149 - 279

MÉDARD J.-F., 1991, États d'Afrique Noire, formation, mécanismes et crises, Paris, Karthala (Études réunies et présentées)

MEZGHANI A., 2011, L’État inachevé, la question du droit dans les pays arabes, Paris, Nrf/ Gallimard

MCEGLIN P., 2005, Outils et médias éducatifs. Une approche communicationnelle, Grenoble, Presses universitaires de Grenoble

MOULINIER-BOUTANG Y., 2007, Le capitalisme Cognitif. La nouvelle grande transformation, Paris, Éditions Amsterdam

MUSSO P., 2011, « Les réseaux amplifient l'action socio-politique, mais ne la remplacent pas » in The Whole World is Watching, New commissions. http://www.ecoledumagasin.com/ 
session21/9pdf/1_pierremusso.pdf. (Dernière consultation le 16 février 2013). Article en ligne sans numérotations

NAJAR S, 2013, Le cyberactivisme au Maghreb et dans le monde arabe, Paris, IRMC/Karthala

NEVEU E., « Médias, mouvements sociaux, espaces publics », Réseaux, 17(98), p.17-85

NEVEU E. (2005, 4e éd.), Sociologie des mouvements sociaux, Paris : La Découverte

NORBERT A., 2013, Donner et prendre, La coopération en entreprise, Paris, La découverte/Poche, 2013

PROULX S., 1999, «L'américanité serait-elle ancrée dans les dispositifs techniques ? » in Florian Sauvageau (dir.), dans Variations sur l'influence culturelle américaine, Québec, Presses de l'Université Laval, pp. 209-230

RIADH F., et MATTELART T., 2011, « Monde arabe : les révolutions 2.0 n'ont pas eu lieu », La Revue-Médias, n³0, pp. 70 - 94

RIEFFEL R., 2014, Révolution numérique, révolution culturelle ?, Paris, Gallimard, Folio actuel

SAVANE V., SARR B.-M., 2012, Y'en a marre : radioscopie d'une jeunesse insurgée au Sénégal, Dakar, L'Harmattan

TINE B, 2014, Les centres d'appels : mode d'emploi, Paris, Editions Publibook

TINE B., SALL A., « Les trajectoires d'emplois des jeunes au Sénégal : entre emplois faute de mieux et projet professionnel ", chapitre pour la rédaction d'un ouvrage collectif sur la crise sociétale au Sénégal (CIERVAL - UGB), à paraitre

TOURAINE A., 1973, La production de la société, Paris, Seuil

VOIROL O., 2005, « Les luttes pour la visibilité. Esquisse d'une problématique », Réseaux, 2005/1 n¹29-130, pp. 89-121

\section{NOTES}

1. Traduction de Céline CANTAT, Doctorante en sociologie- Boursière Marie Curie en 2014-2015 (INTEGRIM).

2. Nous voulons être prudents quant à l'usage de l'appellation de la société civile. Pour ce faire, nous nous appuyons entre autres sur les travaux de Ferrié. Cf., FERRIÉ J-N., 2003, " " Société civile ", autoritarisme et globalisation des normes ", Journal des anthropologues, n94-95, pp.75-91.

3. Wael Ghonim est un jeune égyptien, cyberdissident, très actif dans la révolution égyptienne de 2011 qui a précipité, avec d'autres, la chute du régime Moubarak.

4. Face à la résurgence des délestages de la société nationale d'électricité du Sénégal, des imams ont décidé, en 2008 , de défier le pouvoir en exhortant les populations à ne pas payer leurs factures d'électricité.

5. Le vieux, en Wolof ; terme utilisé pour nommer le Président âgé de 85 ans, mais aussi signe de respect de ces personnes du troisième âge dans la société sénégalaise.

6. Le pays va mal.

7. Le Président Abdou Diouf avait lui-même qualifié cette jeunesse en 1988, de «malsaine ».

8. Le gagnant remporte l'ensemble de la mise.

9. C'est-à-dire une société moins rigide, moins bureaucratique, plus souple avec des relations moins hiérarchiques. 


\section{RÉSUMÉS}

Cette recherche réflexive vise à revenir sur les événements sociopolitiques ayant marqué au début des années 2000 le Sénégal et la Tunisie. Elle vise dans un premier temps à mettre en exergue deux points de césure qui ont caractérisé la littérature : la thèse de la primauté technologique ou encore les TIC génératrices de mouvements sociaux d'une part ; et d'autre part le postulat de l'antériorité sociale c'est-à-dire les TIC comme solubles dans les mouvements sociaux. Cet article interroge, dans un deuxième temps, les manières de faire un mouvement social et la multiplication des médiatisations et de diffusion des idées et des images dans les espaces numériques tels que Facebook, Twitter, You Tube et les Smartphones pour arriver à une position novatrice, médiane et heuristique. Cette articulation est d'autant plus intéressante qu'elle conjugue deux contextes qui sont rarement analysés ensemble dans le champ des sciences sociales à savoir le Sénégal d'un côté - considéré dans la littérature scientifique comme faisant partie des pays africains au sud du Sahara - et la Tunisie étudiée comme faisant partie dudit « monde arabe ». Par cette contribution, nous voulons briser cette catégorisation et montrer la filiation et la possibilité d'élaborer une recherche commune établissant des ponts entre ces deux pays. Notre démarche consiste dès lors à proposer une perspective médiane c'est-à-dire une approche communicationnelle remettant en question les schèmes explicatifs dominants. Elle a pour principal projet, dans un troisième temps de cet article, de dévoiler les enjeux liés à la technologisation de plus en plus repérable des mouvements sociaux.

This research sets out to develop a reflexive analysis of the socio-political events that took place in Senegal and Tunisia in the early 2000s. First, it highlights two rupture points which have marked the academic literature: on the one hand, the argument of the primacy of technology, that is, the idea that ICTs generate social movements and, on the other hand, the hypothesis of the anteriority of social phenomena, which implies that ICTs are merely soluble within social movements. The article then questions the ways in which social movements operate, and in particular the increase in the mediatisation and diffusion of ideas and images through digital sites, such as Facebook, Twitter and You tube, and through Smartphones. This leads to a novel, intermediate and heuristic position, which is all the more interesting as it combines two contexts that have rarely been analyzed together in the social sciences, namely Senegal - considered in academic literature as belonging to sub-Saharan African countries - and Tunisia - considered as part of the so-called "Arab world". We attempt to unsettle this categorization and to show that there exist links and that it is possible to develop common research that builds bridges between the two countries. This research hence questions through empirical analysis the dominant interpretations of the relationship between technologies and social movements. Its main objective, presented in the third section of the article, is to explore what is at stake in the increasingly observable process of technologization of social movements ${ }^{1}$.

\section{INDEX}

Mots-clés : TIC ; changement ; mouvement social ; fétichisation ; réseau numérique ; démocratie Keywords : ICT; social change; social movement; fetishization; social network; democracy 


\section{AUTEURS}

\section{BENOÎT TINE}

Enseignant-chercheur en Sociologie, Université Assane Seck de Ziguinchor, Sénégal et chercheur associé au Laboratoire Printemps/CNRS-UVSQ - France

\section{MOHAMED-ALI ELHAOU}

Docteur en Sciences de la Communication de l'université Paris 13, chercheur en Communication des Organisations à la MSH Paris Nord et chercheur associé à Geriico à Lille 3. Actuellement Maître-assistant à l'Institut de presse et des sciences de l'information à Tunis. 